\title{
Activity from the Be/X-ray binary system V0332+53 during its intermediate-luminosity outburst in 2008
}

\author{
M. D. Caballero-García ${ }^{1}$, A. Camero-Arranz ${ }^{2}$, M. Özbey Arabac1 ${ }^{3}$, C. Zurita ${ }^{4,5}$, J. Suso ${ }^{6}$, J. Gutiérrez-Soto ${ }^{7,8}$,

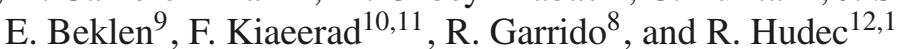

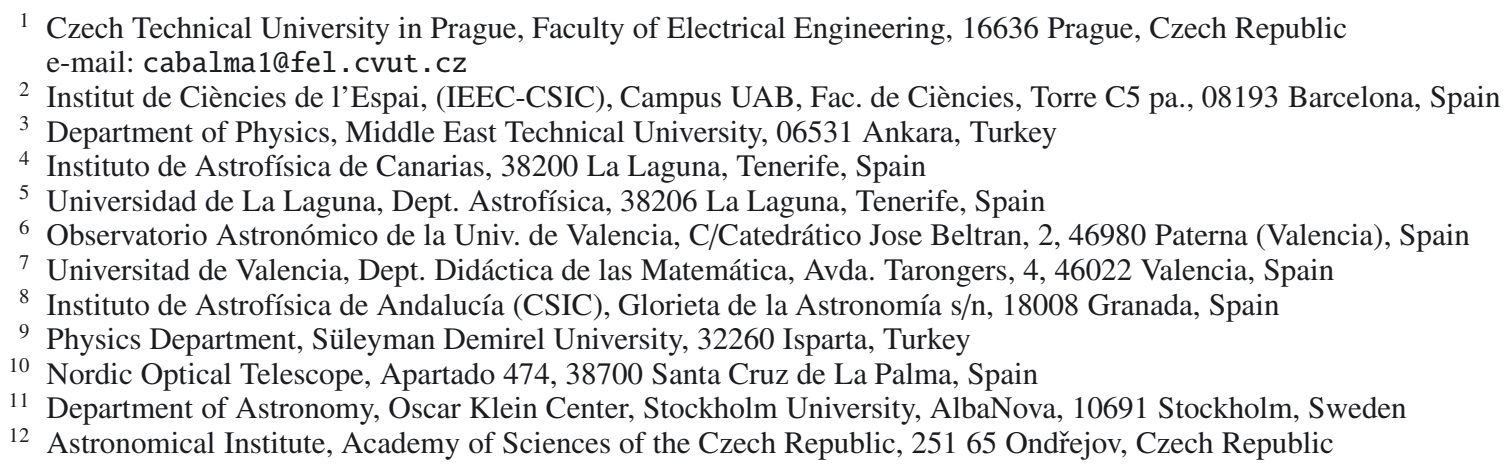

Received 29 June 2015 / Accepted 13 January 2016

\section{ABSTRACT}

\begin{abstract}
Aims. We present a study of the Be/X-ray binary system V $0332+53$ with the main goal of characterizing its behaviour mainly during the intermediate-luminosity X-ray event in 2008. In addition, we aim to contribute to the understanding of the behaviour of the donor companion by including optical data from our dedicated campaign starting in 2006.

Methods. V 0332+53 was observed by RXTE and Swift during the decay of the intermediate-luminosity X-ray outburst of 2008, and with Suzaku before the rising of the third normal outburst of the 2010 series. In addition, we present recent data from the Spanish ground-based astronomical observatories of El Teide (Tenerife), Roque de los Muchachos (La Palma), and Sierra Nevada (Granada), and since 2006 from the Turkish TÜBITTAK National Observatory (Antalya). We have performed temporal analyses to investigate the transient behaviour of this system during several outbursts.

Results. Our optical study revealed that continuous mass ejection episodes from the Be star have been taking place since 2006 and another is currently ongoing. The broad-band $1-60 \mathrm{keV}$ X-ray spectrum of the neutron star during the decay of the 2008 outburst was well fitted with standard phenomenological models that were enhanced by an absorption feature of unknown origin at about $10 \mathrm{keV}$ and a narrow iron K-alpha fluorescence line at $6.4 \mathrm{keV}$. For the first time in V $0332+53$ we tentatively see an increase in the cyclotron line energy with increasing flux (although further and more sensitive observations are needed to confirm this). The fast aperiodic variability shows a quasi-periodic oscillation $(\mathrm{QPO})$ at $227 \pm 9 \mathrm{mHz}$ only during the lowest luminosities, which might indicate that the inner regions surrounding the magnetosphere are more visible during the lowest flux states.
\end{abstract}

Key words. X-rays: binaries - pulsars: general - stars: individual: V 0332+53

\section{Introduction}

Accreting X-ray pulsars are binary systems composed of a donor star and an accreting neutron star (NS). In high-mass X-ray binary (HMXB) systems the optical companion could be either a massive early-type supergiant (supergiant systems) or an $\mathrm{O}$, B main-sequence or giant star (BeX binaries; BeXB). Among the most remarkable signatures found in $\mathrm{BeXBs}$ are the detection of IR excess and emission-line features in their optical spectra produced in a disc-like outflow around the Be star. Historically, their outbursts have been divided into two classes. Type I (or normal) outbursts normally peak at or close to the periastron passage of the NS $\left(L_{X} \leq 10^{37} \mathrm{erg} \mathrm{s}^{-1}\right)$. Type II (or giant) outbursts reach luminosities of the order of the Eddington luminosity ( $L_{X} \sim 10^{38} \mathrm{erg} \mathrm{s}^{-1}$; Frank et al. 2002), with no preferred orbital phase. We will refer to any X-ray outburst that does not have these properties as "intermediate" $\left(L_{X} \sim 10^{37}-\right.$ $10^{38} \operatorname{erg~s}^{-1}$ ).

\section{1. $\vee 0332+53$}

The recurrent hard X-ray transient V 0332+53 was detected with the Vela 5B observatory in 1973 during its giant outburst, reaching a peak intensity of $\approx 1.6 \mathrm{Crab}$ in the $3-12 \mathrm{keV}$ energy band (Terrell \& Priedhorsky 1984). The system had gone through a ten-year X-ray quiescent phase when $4.4 \mathrm{~s}$ pulsations were detected with Tenma and EXOSAT satellites (Stella et al. 1985). These X-ray activities, a series of Type I outbursts, lasted about three months separated by the orbital period (34.25 d) of the system. During these outbursts, rapid random fluctuations in the $\mathrm{X}$-ray emission in addition to the pulse-profile variations were also reported (Unger et al. 1992). The system underwent another outburst in 1989 and classified as Type II, which led to the discovery of a cyclotron line scattering feature (CRSF) at $28.5 \mathrm{keV}$ and two quasi-periodic oscillations (QPOs) centred at $0.051 \mathrm{~Hz}$ and $0.22 \mathrm{~Hz}$ (Makishima et al. 1990; Takeshima et al. 1994; Qu et al. 2005). 
The optical companion of the system, BQ Cam (Argyle et al. 1983), is an O8-9-type main-sequence star at a distance of $\approx 7 \mathrm{kpc}$ (Negueruela et al. 1999). It has been widely observed both in the optical and IR wavelengths since its identification. The optical spectrum is characterized by the highly variable emissions of $\mathrm{H}_{\alpha}, \mathrm{H}_{\beta}$, and $\mathrm{H}_{\gamma}$ in addition to the He I lines. The photometric data shows an IR excess (Bernacca et al. 1984; Coe et al. 1987; Honeycutt \& Schlegel 1985; Unger et al. 1992). The brightening in the optical/IR light curves is usually accompanied by the X-ray outburst phases as in the case of the giant 2004 outburst of the system. Goranskij \& Barsukova (2004) predicted this outburst based on the optical brightening of V 0332+53 in the optical/IR band. During the outburst, three additional CRSFs at 27,51 , and $74 \mathrm{keV}$ were detected in Rossi X-ray Timing Explorer (RXTE) observations (Coburn et al. 2005) and confirmed by the subsequent INTEGRAL data (Pottschmidt et al. 2005). Tsygankov et al. (2006) showed that the energy of these features are linearly anti-correlated with its luminosity. The subsequent X-ray activities of the system were in 2008, 2009, and 2010 with relatively weaker peak fluxes (Krimm et al. 2008, 2009; Nakajima et al. 2010). The system was in X-ray quiescence until 18 June 2015, when the Be companion most probably reached its maximum optical brightness after renewing activity in 2012 (Camero-Arranz et al. 2015).

Here, we present a multiwavelength study of $\mathrm{V} 0332+53$ mostly during the recent events initiated in 2008. For this purpose we used archived Swift/XRT and RXTE pointed observations carried out in 2008, one Suzaku observation from 2010, and survey data from different space-borne telescopes that covered intermediate and low-luminosity events. In addition, we used optical/IR data from our dedicated campaign involving several ground-based astronomical observatories. This provides us a unique opportunity to undertake studies of X-ray outbursts of intermediate and low X-ray luminosities. The latter is a regime very scarcely explored in $\mathrm{V} 0332+53$, one of the BeX with the strongest magnetic field known.

\section{Observations and data reduction}

\subsection{Optical/IR photometric observations}

The optical counterpart was observed in the optical band with the $80 \mathrm{~cm}$ IAC80 telescope at the Observatorio del Teide (OT; Tenerife, Spain) from August 2014 until February 2015 (see Supplementary material Table A.1 and Fig. 1). We obtained the optical photometric CCD images using $B$ and $V$ filters with an integration time of $60 \mathrm{~s}$. In addition, on 20 December 2014 a single infrared $J, H$, and $K_{\mathrm{s}}$ observation was performed by the CAIN camera from the $1.5 \mathrm{~m}$ TCS telescope at the OT, with integration times of 150,90 , and $90 \mathrm{~s}$, respectively. The reduction of the data was performed by using the pipelines of both telescopes based on the standard aperture photometry (Camero et al. 2014, for more details on reduction).

The main part of the long-term optical CCD observations of $\mathrm{V} 0332+53$ include data from the $0.45 \mathrm{~m} \mathrm{ROTSEIIId}^{1}$ telescope from the TÜBITAK National Observatory (TUG; Antalya, Turkey) between February 2006 and December 2012. This telescope operates without filters and is equipped with a $2048 \times$ 2048 pixel CCD. Dark and flat-field corrections of all images were done automatically by a pipeline. Instrumental magnitudes

\footnotetext{
The Robotic Optical Transient Search Experiment (ROTSE) is a collaboration of Lawrence Livermore National Lab, Los Alamos National Lab, and the University of Michigan (http://www . ROTSE . net).
}

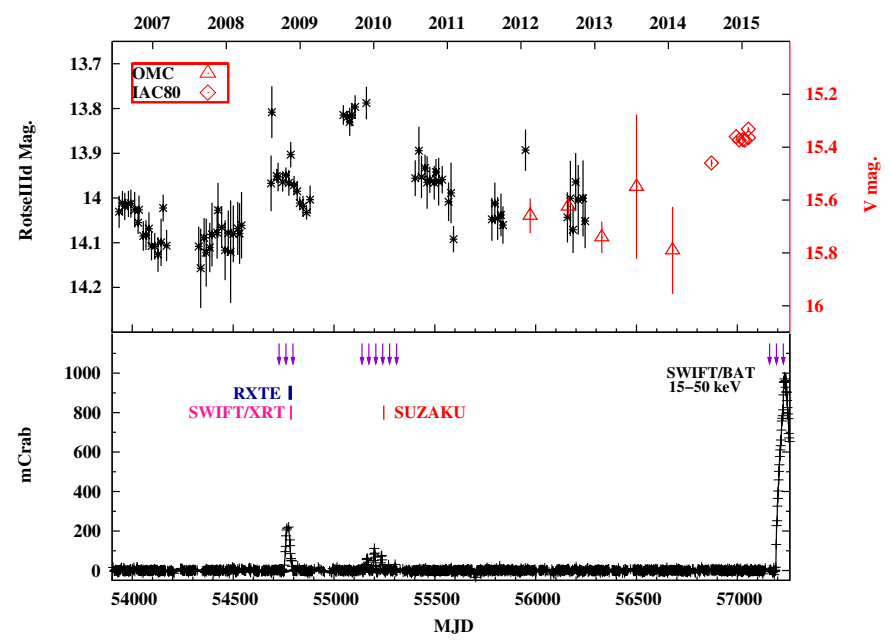

Fig. 1. Comparison of the Swift/BAT light curve (15-50 keV) with the optical magnitudes (ROTSE, OMC, IAC80) of V 0332+53 since 2006. The BAT, ROTSE, and OMC data points have been rebinned for clarity. The violet arrows in the X-ray panel represent the times of the periastron passages of the NS.

of all the corrected images were obtained using the SExtractor Package (Bertin \& Arnouts 1996). Calibrated ROTSEIIId magnitudes were acquired by comparing all the stars in each frame with the USNO-A2.0 catalogue $R$-band magnitudes.

In addition, we used data from the Optical Monitoring Camera (OMC; Mas-Hesse et al. 2003) on board the INTEGRAL satellite (Winkler et al. 2003). They were obtained from the Data Archive Unit at CAB Astrobiology centre ${ }^{2}$ (INTA-CSIC), and pre-processed by the INTEGRAL Science Data Centre (ISDC) ${ }^{3}$.

\subsection{Optical spectroscopic observations}

Optical spectroscopic observations of the donor star were performed from 26 September 2006 until 22 November 2014 (see Supplementary material Table A.2) with three different telescopes: the Russian-Turkish $1.5 \mathrm{~m}$ telescope (RTT150) at the TUG, the $2.56 \mathrm{~m}$ Nordic Optical Telescope (NOT) located at the Observatorio del Roque de los Muchachos (La Palma, Spain), and the $1.5 \mathrm{~m}$ Telescope at the Observatorio de Sierra Nevada (OSN-CSIC) in Granada (Spain).

The spectroscopic data from RTT150 were obtained with the TÜBITAK Faint Object Spectrometer and Camera (TFOSC). The reduction of the spectra was done using the Long-Slit package of MIDAS ${ }^{4}$. The low-resolution OSN spectra were acquired using Albireo spectrograph, whereas the NOT spectra were obtained with the Andalucía Faint Object Spectrograph and Camera (ALFOSC). The reduction of this data set was performed using standard procedures within IRAF $^{5}$ (see Camero et al. 2014 for further details on the instrumentation and data reduction).

All spectroscopic data were normalized with a spline fit to continuum and corrected to the barycentre after the wavelength

\footnotetext{
http://sdc.cab.inta-csic.es/omc/index.jsp

http://www.isdc.unige.ch

4 http://www.eso.org/projects/esomidas

5 IRAF is distributed by the National Optical Astronomy Observatory, which is operated by the Association of Universities for Research in Astronomy (AURA) under cooperative agreement with the National Science Foundation.
} 
Table 1. Log of the X-ray observations.

\begin{tabular}{lcccll}
\hline \hline Obs. & Date $^{a}$ & MJD & Telescope & $\begin{array}{l}\text { Exp. } \\
\text { time } \\
\text { (s) }\end{array}$ & $\begin{array}{l}\text { Pulse } \\
\text { period } \\
\text { (s) }\end{array}$ \\
\hline 1 & $04 / 11 / 08$ & 54774.636 & RXTE $^{b}$ & 1600 & $4.3742(1)$ \\
2 & $07 / 11 / 08$ & 54777.999 & RXTE $^{\prime}$ & 752 & - \\
3 & $09 / 11 / 08$ & 54779.731 & RXTE & 496 & - \\
4 & $10 / 11 / 08$ & 54780.594 & RXTE & 864 & - \\
5 & $12 / 11 / 08$ & 54782.394 & RXTE & 1456 & $4.3740(1)$ \\
6 & $14 / 11 / 08$ & 54784.511 & RXTE & 1552 & $4.3746(6)$ \\
7 & $16 / 11 / 08$ & 54786.543 & RXTE & 1488 & $4.37450(2)$ \\
8 & $19 / 11 / 08$ & 54789.826 & RXTE & 3840 & $4.37540(2)$ \\
9 & $20 / 11 / 08$ & 54790.775 & RXTE & 2848 & $4.3763(1)$ \\
10 & $21 / 11 / 08$ & 54791.743 & RXTE & 1152 & $4.3760(1)$ \\
11 & $22 / 11 / 08$ & 54792.270 & RXTE & 3716 & $4.37610(4)$ \\
12 & $23 / 11 / 08$ & 54793.273 & RXTE & 1597 & $4.37620(4)$ \\
13 & $23 / 11 / 08$ & 54793.387 & RXTE & 3235 & $4.37620(5)$ \\
\hline$(5)$ & $12 / 11 / 08$ & 54782.384 & Swift & 1706 & - \\
$(6)$ & $14 / 11 / 08$ & 54784.520 & Swift & 2108 & - \\
$(7)$ & $16 / 11 / 08$ & 54786.533 & Swift & 2307 & - \\
$(8)$ & $19 / 11 / 08$ & 54789.338 & Swift & 1613 & - \\
$(9)$ & $20 / 11 / 08$ & 54790.811 & Swift & 1597 & - \\
$(10)$ & $21 / 11 / 08$ & 54791.487 & Swift & 1489 & - \\
\hline 14 & $16 / 02 / 10$ & 55243.264 & Suzaku $^{d}$ & 15946 & $4.37(1)$ \\
\hline
\end{tabular}

Notes. Standard mode for RXTE and Suzaku and Windowing mode for Swift/XRT. (a) Observation date in the format (dd/mm/(20)yy). (b) RXTE/PCA exposure time. (c) Swift/XRT exposure time. (d) Suzaku/XIS1 exposure time.

calibration. The full width at half maximum (FWHM) and equivalent width (EW) measurements of $\mathrm{H}_{\alpha}$ lines were acquired by fitting Gaussian functions to the emission profiles using the ALICE subroutine of MIDAS

\subsection{X-ray observations}

The XRT (onboard the Swift satellite; Gehrels et al. 2004) observed V $0332+53$ in Windowing Timing (WT) mode $(1.7 \mathrm{~ms}$ time resolution, 1D Image) six times from 12 to 21 November 2008. Table 1 shows a summary of the log of these observations. To reduce the data and extract the final products (spectra and light curves) we followed the standard recipe as detailed in the Swift/XRT data reduction guide ${ }^{6}$. The relevant response matrix to use is given by the HEASARC calibration database $\left(\mathrm{CALDB}^{7}\right)$. All the XRT light curves were barycentre corrected. For comparison we also used data products supplied by the UK Swift Science Data Centre at the University of Leicester (Evans et al. 2009).

RXTE Proportional Counter Array (PCA; Jahoda et al. 1996) standard 1 and 2 data only from PCU-2 were selected for this work. To extract PCA products we followed the standard procedure showed in its cookbook ${ }^{8}$. A systematic error of $0.5 \%$ was added in quadrature to the PCA standard 2 spectra, as recommended by the instrument team. Products from the High Energy X-ray Timing Experiment (HEXTE; Gruber et al. 1996)

\footnotetext{
6 http://swift.gsfc.nasa.gov/analysis/xrt_swguide_v1_ 2.pdf

7 http://heasarc.gsfc.nasa.gov/FTP/caldb

8 https://heasarc.gsfc.nasa.gov/docs/xte/recipes/pca_ spectra.html
}

were also extracted using the corresponding cookbook recipe ${ }^{9}$. Only standard mode data from cluster B were used. All the RXTE light curves were barycentre corrected.

In addition, we analysed one observation performed by Suzaku (Mitsuda et al. 2007) on 16 February 2010. We selected data from the X-ray Imaging Spectrometer (XIS; Koyama et al. 2007) CCD and the PIN diode detector (10-70 keV) of the Hard X-ray Detector (HXD; Takahashi et al. 2007). For this observation only the XIS0, XIS1, and XIS3 detectors were available. Following the Suzaku ABC guide ${ }^{10}$, we reprocessed all the data and extracted the final products. The clocking mode option was normal burst (1.0) with the $1 / 4$ window selected ( $1 \mathrm{~s}$ time binning), and a data editing mode of $3 \times 3$. No pile-up correction was needed. The events were barycentre corrected.

We used the HEAsoft software version 6.15.1 (Arnaud 1996) for the data analysis of all the instruments. Since 2008, the Gamma-ray Burst Monitor (GBM) on board the Fermi satellite has been monitoring V $0332+53$. In this work we used timing products provided by the GBM Pulsar Team ${ }^{11}$ (see e.g. Finger et al. 2009; Camero-Arranz et al. 2010, for a detailed description of the timing technique). We also used quick-look X-ray results provided by the RXTE All Sky Monitor team ${ }^{12}$, and since 2008 we used the quick-look X-ray Swift/BAT transient monitor results provided by the Swift/BAT team ${ }^{13}$ (Krimm et al. 2013; see Fig. 2).

\section{Results}

\subsection{Optical photometry}

The optical light curves of BQ Cam compared to the Swift/BAT data (15-50 keV) from June 2006 to June 2015 are shown in Fig. 1. In 2008, after being $\approx 3.5 \mathrm{yr}$ in quiescence in $\mathrm{X}$-rays, the system underwent a new active period. However, the optical companion entered the brightening state nearly $1.5 \mathrm{yr}$ before the NS. At this time the enhancement in the optical brightness reached a value of $\approx 0.25 \mathrm{mag}$. The $\mathrm{X}$-ray outburst was triggered around 17 October 2008 (MJD 54 756) roughly six days before the periastron passage of the NS (see Fig. 1). This large flare lasted about $40 \mathrm{~d}$ and reached a flux of $\approx 214 \mathrm{mCrab}$ within 3 weeks. It should be pointed out that the optical magnitude of V $0332+53$ shows a sudden decrease when the X-ray outburst declines. Fading of the optical companion continued until the end of January 2009 (MJD $\approx 54850$ ) and soon after it turned back to its brightening state. The X-ray flux, on the other hand, stayed at its quiescent level until the Be star reached a peak value of $\approx 13.8 \mathrm{mag}$ (ROTSE magnitude). A new X-ray phase of the system started in November 2009, again a few days after the periastron passage (MJD $\approx 55140$ ) of the NS. That activity included five small but periodic outbursts separated by the orbital period (34.25d) of the system classified as Type I.

The second outburst of this series was the most powerful one with a peak flux of $\approx 110$ mCrab. The optical magnitude, on the other hand, started to decrease with the decline of the second outburst $(\mathrm{MJD} \approx 55201)$. The X-ray activities finished by the end of May 2010 (MJD $\approx 55312$ ) while the optical magnitude was still fading. It ceased around August 2011 (MJD $\approx 55780$ )

\footnotetext{
9 https://heasarc.gsfc.nasa.gov/docs/xte/recipes/ hexte.html

${ }^{10}$ http://heasarc.nasa.gov/docs/suzaku/analysis/abc

${ }^{11}$ http://gammaray.nsstc.nasa.gov/gbm/science/pulsars

12 http://xte.mit.edu/ASM_lc.html

13 http://swift.gsfc.nasa.gov/results/transients
} 


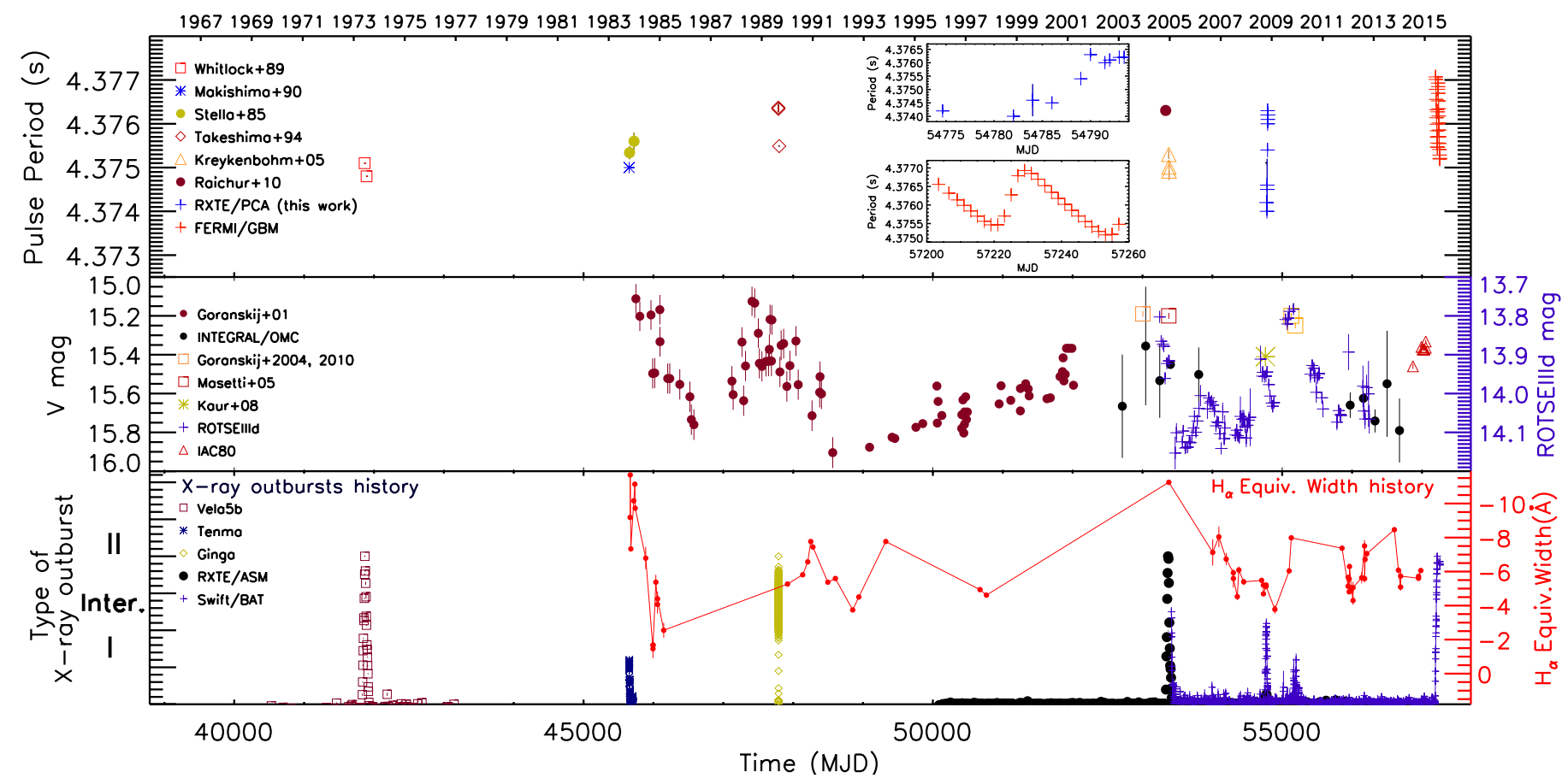

Fig. 2. Top: long-term pulse period history of V $0332+53$ since its discovery in 1973. Middle: Long-term optical light curve of V $0332+53$. All but the IAC80 light curve have been rebinned for clarity. Bottom: type of X-ray outburst undergone by V 0332+53 (i.e. Type II, Intermediate, and Type I). The intensities are the same for outbursts of the same type and have not been corrected for the different energy bands. We only intend to be illustrative of the times and types of events. The long-term evolution of the $\mathrm{H}_{\alpha} \mathrm{EW}$ (red filled circles) is also overplotted. The measurements have been extracted from Iye \& Kodaira (1985), Kodaira et al. (1985), Corbet et al. (1986), Negueruela et al. (1999), Masetti et al. (2005), Kiziloglu et al. (2008), Goranskij et al. (2010), and the present work.

and lasted $\approx 4$ years. Our recent data from the IAC80 telescope and the optical camera OMC on board INTEGRAL shows an optical enhancement of the counterpart BQ Cam; the brightness varies from the quiescence level of $V=15.93 \pm 0.55$ (6 January 2012; MJD 55 932.60) to $V=15.332 \pm 0.008$ (1 February 2015; MJD 57 054.04; Camero-Arranz et al. 2015). This corresponds to an increment of $\approx 0.6 \mathrm{mag}$, similar to that observed during the brightening episodes that occurred in 1983 (see Goranskij 2001) and in 2004 (Goranskij \& Barsukova 2004), but higher than the episodes in 2008 (Kaur et al. 2008) and 2009 (Goranskij et al. 2010). This is confirmed by our only IR measurement from December 2014 (MJD 57 011.869), which showed an unusually bright $\mathrm{Be}$ companion. The magnitudes obtained were $J=11.228 \pm 0.003 \mathrm{mag}, H=10.651 \pm 0.003 \mathrm{mag}$, and $K_{\mathrm{s}}=10.285 \pm 0.004 \mathrm{mag}$, which are comparable to those from December 1983 (Williams et al. 1983). We note that BQ Cam did not reach its maximum level $(V=15.2 \mathrm{mag})$ during our observations from February 2015. The maximum has probably been reached recently, since the BAT monitor is currently detecting $\mathrm{X}$-ray activity from $\mathrm{V} 0332+53$ with a daily average flux of the order of 310.4 mCrab on 25 June 2015 (MJD 57 198.0).

\section{2. $H_{\alpha}$ line}

We studied the long-term $\mathrm{H}_{\alpha}$ line monitoring of BQ Cam from September 2006 to November 2014 (see Fig. 3). In contrast to the previous works stating the $\mathrm{H}_{\alpha}$ profile variations (Negueruela et al. 1998, 1999), we did not see such variability patterns during the observation period. Instead, all the line profiles are nearlysymmetric in a single-peaked form despite the lack of observations for the period 2010-2011.
Although it has the same single-peaked emission profile on a long-term basis, the $\mathrm{H}_{\alpha}$ line strength does not show a constant trend during the spectroscopic observations (see Supplementary material Table A.2). In Fig. 4 the evolution of EW and FWHM measurements of the emission line are shown. The first two measurements of the line coincide with the declining phase of the optical outburst seen in the time interval MJD 53 576-54 180. The EW values decreased as the star faded away, which can be attributed to the weakening of the decretion disc; this decreasing pattern continued after the optical outburst triggered (MJD 54 324). Although our data coverage is not uniform, we see a reverse relation between the optical magnitude and the width of the emission line until 4 March 2009 (MJD 54894) when the lowest value of the $\mathrm{EW}$ of $\mathrm{H}_{\alpha}$ is reached (see Fig. 2). After about seven months, the EW reached a value of $-5.21 \AA$. Although this sudden increase in the line width well fits with the rising of the optical magnitude, we cannot make any further explanation regarding the behaviour of the emission line because of the lack of data for 2010-2011. From 2012 to 2014, we see that the strength of the $\mathrm{H}_{\alpha}$ line kept its varying pattern without a noticeable change in the optical magnitude which would have indicated the upcoming mass ejection event.

In contrast to the variations in EW values, the FWHM values stayed approximately constant until the end of 2012. At that point, the FWHM values started to follow nearly the same pattern of EW. Indeed, it is not unusual for EW and FWHM to show a similar behaviour since the width of the emission profile is directly related to the rotational velocity of the Be star. Hanuschik (1989) gives the relation between the $\mathrm{H}_{\alpha}$ widths and the projected rotational velocity, $v \sin (i)$. The projected rotational velocity of $\mathrm{V} 0332+53$ is found to be $v \sin (i)=166.68 \mathrm{~km} \mathrm{~s}^{-1}$, using the average values of $-5.04 \AA$ 

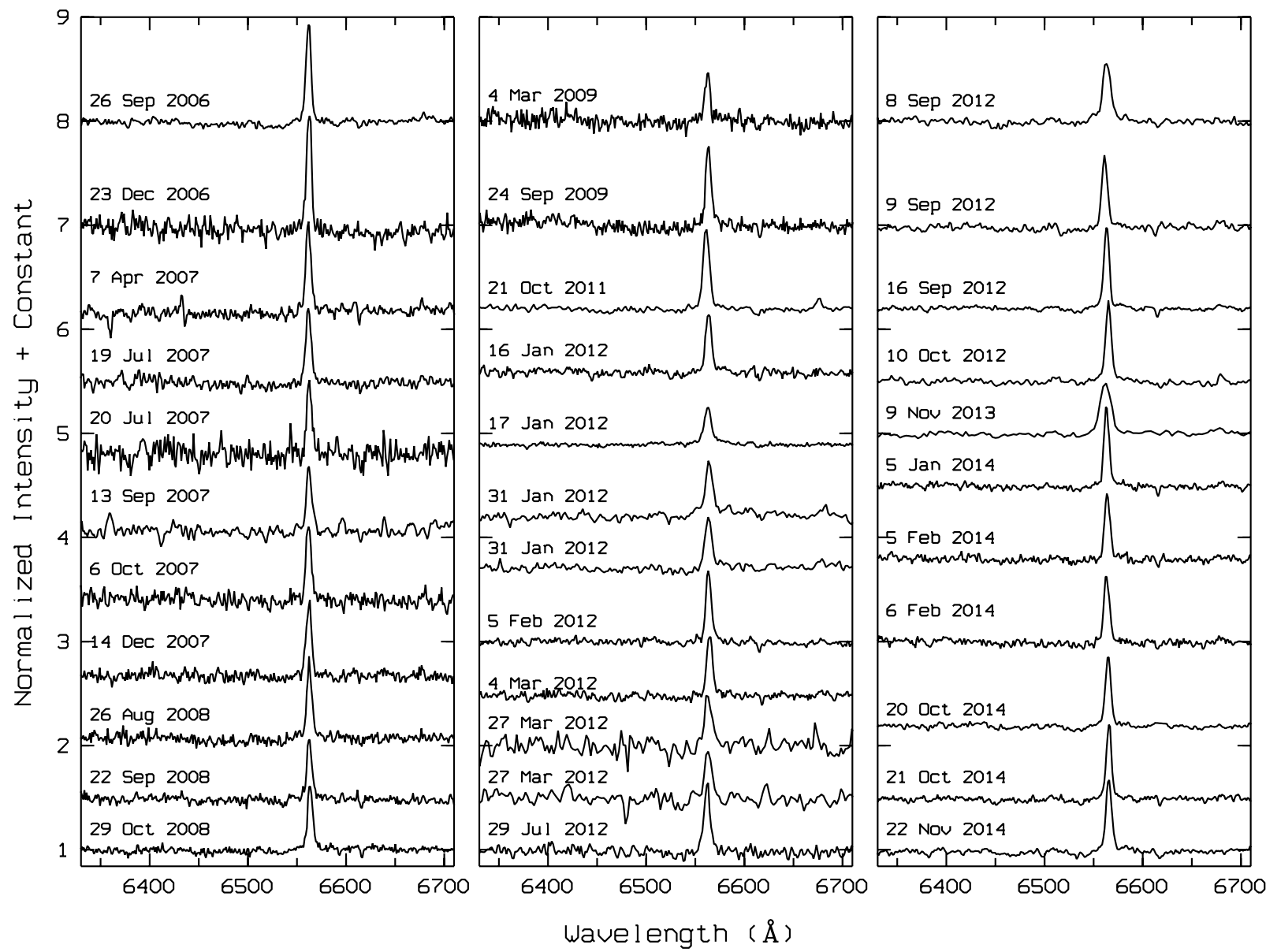

Fig. 3. $\mathrm{H}_{\alpha}$ profiles of $\mathrm{V} 0332+53$ selected between 2006 and 2014. The single-peaked emission of the line does not change on a long-term basis.

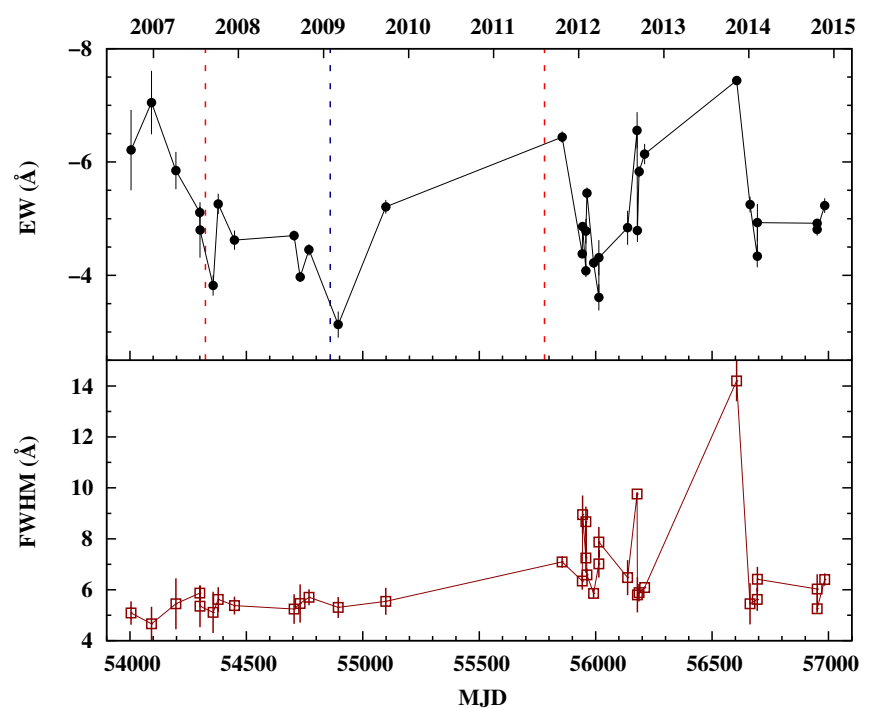

Fig. 4. Evolution since 2006 of the EW and FWHM measurements of the $\mathrm{H}_{\alpha}$ emission line. The red dashed vertical lines denote the beginning and the end of the optical outburst seen in ROTSEIIId light curve (see also Fig. 1), whereas the black dashed line shows the time of the turning point $(\approx 10 \mathrm{~d})$ from decreasing to increasing trend just after the strong $\mathrm{X}$-ray outburst around MJD $\approx 54850$.

(i.e. $\left.230 \mathrm{~km} \mathrm{~s}^{-1}\right)$ and $7.3 \AA\left(333.56 \mathrm{~km} \mathrm{~s}^{-1}\right)$ for the EW and FWHM, respectively (see Supplementary material Table A.2). For the true rotational velocity of $\mathrm{V} 0332+53$, we have assumed the inclination angle of the system of $i \leq 18.9^{\circ}$ as suggested by Zhang et al. (2005). Therefore, the lower limit to the true rotational velocity would be $514 \mathrm{~km} \mathrm{~s}^{-1}$. Using the typical mass and radius values for a late O-type $\operatorname{star}\left(M \gtrsim 20 M_{\odot}\right.$ and $\left.R \gtrsim 15 R_{\odot}\right)$, the break-up velocity is determined to be $600 \mathrm{~km} \mathrm{~s}^{-1}$, which is close to the rotational velocity.

\subsection{X-ray activity}

\subsubsection{Periodic timing analysis}

To search for periodic pulsations from the compact object we used the tool efsearch from the Xronos ${ }^{14}$ package. This tool searches for periodicities in a time series by folding the data over a range of periods and by searching for a maximum chi-square as a function of period. Standard 1 PCA, XRT, and Suzaku-XIS barycentred light curves for $\mathrm{V} 0332+53$ were selected for this task (see Table 1). The resulting pulse periods can be seen in Fig. 2 and Table 1. The error on the measured spin periods is calculated using the chi-square versus spin period plot provided by efsearch. The points near the peak of this plot are fitted with a Gaussian. The error on the Gaussian centre estimate is taken as the error on the spin period. From Table 1 we can see that during the 2008 outburst decay the neutron star seems to spin more slowly, as expected. Additionally, we note that in Fig. 2 we do not include the pulse period obtained with Suzaku-XIS $\left(P_{\text {pulse }}=4.37(1) \mathrm{s}\right)$ because of its large uncertainty. Furthermore,

\footnotetext{
14 http://heasarc.gsfc.nasa.gov/docs/xanadu/xronos/ xronos.html
} 
Table 2. Results from the RXTE timing analysis.

\begin{tabular}{lccccc}
\hline \hline & Obs. 1 & Obs. 2 & Obs. 6 & Obs. 8 & Obs. 9 \\
\hline$v_{1}$ & $7.5 \pm 7$ & - & - & $10 \pm 8$ & $12 \pm 10$ \\
$F W H M$ & $174 \pm 14$ & - & - & $29 \pm 14$ & $22 \pm 20$ \\
Norm. & $21.7 \pm 1.2$ & - & - & $1.7 \pm 0.8$ & $1.1 \pm 0.6$ \\
rms $(\%)$ & $16.4 \pm 0.4$ & - & - & $6.2 \pm 0.3$ & $8.3 \pm 0.6$ \\
\hline$v_{2}$ & - & $48 \pm 13$ & $36 \pm 11$ & $82 \pm 19$ & $77 \pm 25$ \\
$F W H M$ & - & $147 \pm 15$ & $166 \pm 12$ & $137 \pm 25$ & $160 \pm 24$ \\
Norm. & - & $17.2 \pm 1.5$ & $9.1 \pm 0.5$ & $1.3 \pm 0.4$ & $1.3 \pm 0.4$ \\
rms $(\%)$ & - & $16.4 \pm 0.7$ & $16.5 \pm 0.5$ & $6.5 \pm 0.3$ & $8.4 \pm 0.6$ \\
\hline$v_{\text {QPO }}$ & - & - & $231 \pm 5$ & $226 \pm 8$ & $233 \pm 4$ \\
$F W H M$ & - & - & $16 \pm 11$ & $33 \pm 18$ & $17 \pm 16$ \\
Norm. & - & - & $0.46 \pm 0.15$ & $0.16 \pm 0.06$ & $0.12 \pm 0.04$ \\
rms $(\%)$ & - & - & $3.8 \pm 2.1$ & $2.3 \pm 1.0$ & $2.7 \pm 1.8$ \\
\hline$\Gamma_{\text {Poisson }}$ & 0 & 0 & 0 & 0 & 0 \\
$N_{\text {Poisson }}$ & $1.84 \pm 0.04$ & $1.59 \pm 0.05$ & $1.79 \pm 0.03$ & $1.90 \pm 0.03$ & $1.93 \pm 0.03$ \\
\hline$\chi^{2} / v$ & $0.84(213 / 252)$ & $1.0(253 / 252)$ & $0.98(243 / 249)$ & $0.99(244 / 246)$ & $0.80(196 / 246)$ \\
\hline
\end{tabular}

Notes. Model used: lorentz+lorentz+lorentz+powerlaw for five RXTE/PCA observations. The PDS were created in the (2-60) keV energy band and $\left(8 \times 10^{-3}-4\right) \mathrm{Hz}$ frequency range. The frequency and width $(v$ and FWHM) of the components are shown in units of mHz. Errors are $68 \%$ confidence errors.

we do not include any pulse period from the XRT data because they were not significantly detected.

\subsubsection{Aperiodic timing analysis}

We performed an analysis of the fast time variability of $\mathrm{V} 0332+53$ in the $2-60 \mathrm{keV}$ energy range of Obs. $1,2,6,8$, and 9 ; the net PCA count rate is $\approx 700,600,300,300,160 \mathrm{cts} \mathrm{s}^{-1}$, respectively. The rest of the observations are too short and/or the source is too faint to perform timing analysis. The time resolution of the PCA in the mode used is $0.125 \mathrm{~s}$.

We used the GHATS package, developed under the IDL environment at INAF-OAB ${ }^{15}$, to produce the power density spectra (PDS) from 512 points in each light curve. The PDS were then averaged together for each observation. The PCA light curve was binned at its time resolution $(0.125 \mathrm{~s})$. This yields a Nyquist frequency of $=4 \mathrm{~Hz}$. The PDS were normalized according to Leahy et al. (1983). All of the PDS show low-frequency band-limited noise (which may turn into peaked noise) and QPO noise.

Power density spectra fitting was carried out with the standard XSPEC fitting package by using a unit response. Fitting the $(2-60 \mathrm{keV})$ PDS with a model constituted by a zero centred Lorentzian for the flat-topped noise (hereafter $L_{1}$ ) plus a constant for the Poissonian noise (i.e. well reproduced by a powerlaw model component with its photon index frozen to zero in the fits) results in mostly acceptable chi-square values only for the PDS of Obs. 1 (see Table 2). In the cases of Obs. 8 and 9 the fit statistics with this model is worse and leaves some visible residuals, i.e. $\chi^{2} / v=290 / 252,233 / 252$, respectively. The fit to these observations improved by adding a second Lorentzian component $\left(\mathrm{L}_{2}\right)$ centred at $v=30-80 \mathrm{mHz}$. This component changed the F-test fits statistics by $\Delta \chi^{2} \approx 20-30$ for $v=249$ degrees of freedom (d.o.f.), thus a $\leq 3 \sigma$ improvement. For Obs. 2 and 6 we only took into account the latter feature since it is broad and therefore constitutes a main part of the measure of the root mean square (rms), i.e. by $(10-20) \%$. This resulted in a fit statistics of $\chi^{2} / v=253 / 252,255 / 252$ for Obs. 2 and 6 , respectively.

\footnotetext{
${ }_{15}$ http://www.brera.inaf.it/utenti/belloni/GHATS_ Package/Home.html
}

The fit of the $(2-60 \mathrm{keV})$ PDS with a model constituted by a zero $\left(\mathrm{L}_{1}\right)$ and a $v=30-80 \mathrm{mHz}\left(\mathrm{L}_{2}\right)$ centred Lorentzians $\left(\mathrm{L}_{2}\right.$ only for Obs. 2 and 6) for the band-limited noise plus a constant for the Poissonian noise results in a poor description of the data for Obs. 6, 8, and 9; the fit statistics with this model is of $\chi^{2} / v=255 / 252,263 / 249,209 / 249$, respectively. In these observations there are positive residuals centred in the range of (220-240) $\mathrm{mHz}$ that we fitted by adding a further Lorentzian component $\left(L_{\mathrm{QPO}}\right)$ centred at those frequencies. This component changed the fits statistics by $\Delta \chi^{2} \approx 12,19,13$ for $v=249,246,246$ d.o.f., thus a $(2.9,3.9,3.0) \sigma$ improvement for Obs. 6, 8, and 9, respectively. We took into account this feature, since it is broad and therefore substantially affects the measure of the rms, i.e. by $\approx(2-4) \%$ for all the observations. These positive features have a peaked form, thus we will call them QPOs, consistent with previous findings (Qu et al. 2005; Reig 2008; Reig $\&$ Nespoli 2013). The detection of this peak is only significant for Obs. 8, which can be explained since this is the observation with the longest exposure time of the sample.

We calculated the fractional rms from the best-fit model (integrated in the $\left(8 \times 10^{-3}-4\right) \mathrm{Hz}$ band). This was found to be $(15-20) \%$ in the $2-60 \mathrm{keV}$ energy range. The results obtained from the global fits (i.e. using the lorentz+lorentz+lorentz+powerlaw model in XSPEC) are given in Table 2. In Fig. 5 we plot the broad-band PDS with the best-fit model. We note that our results are broadly in agreement with those previously obtained (Reig \& Nespoli 2013).

\subsubsection{Spectral analysis}

We fitted the brightest background-subtracted spectra with standard spectral models in XSPEC 12.8.2 (Arnaud 1996). All errors quoted in this work are $68 \%(1 \sigma)$ confidence. The spectral fits were limited to the $1-10,4.5-28,26-60 \mathrm{keV}$ range for the XRT, PCA, and the HEXTE, respectively, where the calibration of the instruments is the best. The spectra were grouped in order to have at least 100, 50, and 50 counts for the XRT, PCA and HEXTE, respectively, for each background-subtracted spectral channel and to avoid oversampling of the intrinsic energy resolution. 


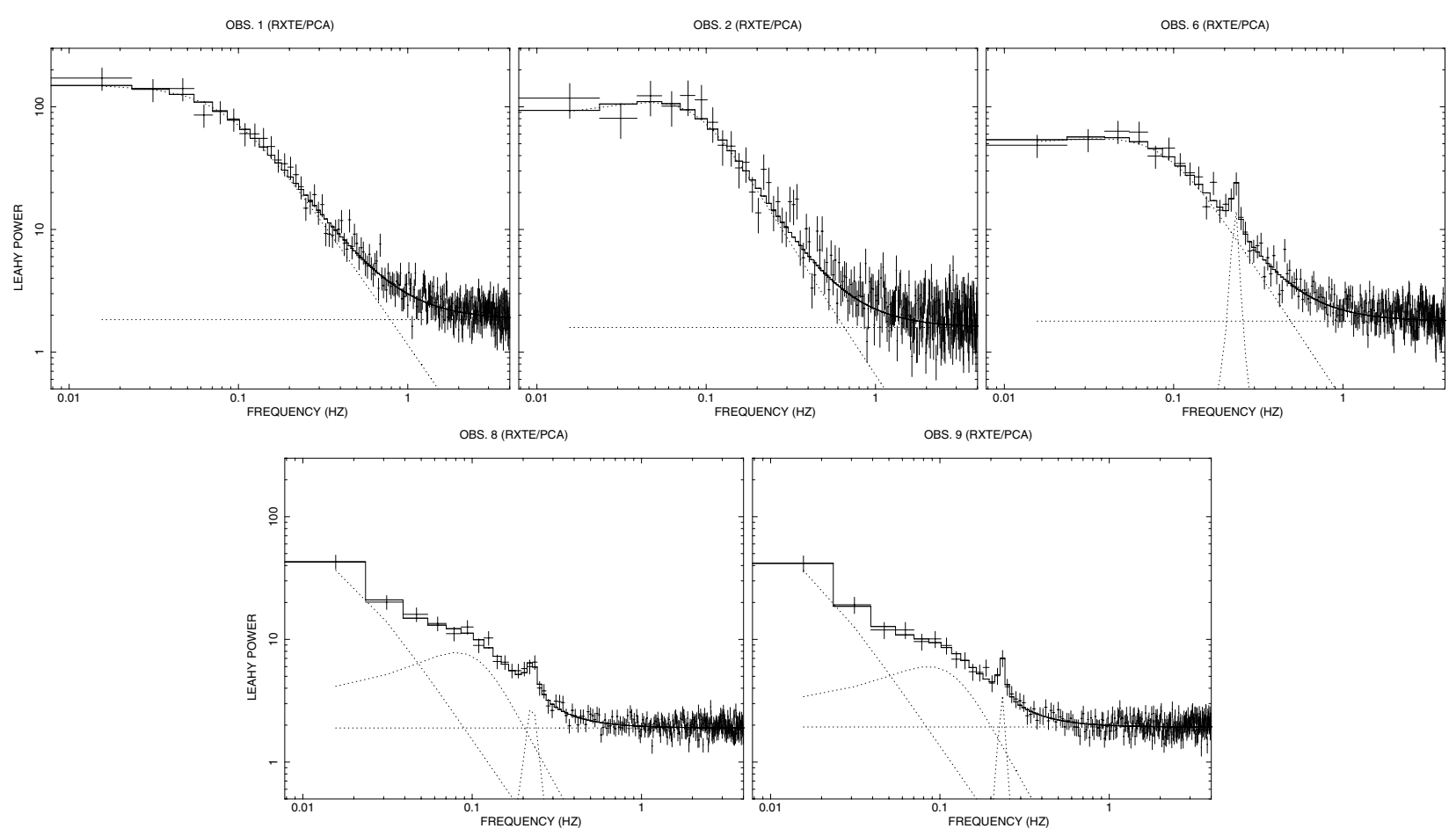

Fig. 5. Power density spectra of the RXTE PCA data in the energy and frequency range $(2-60 \mathrm{keV})$ and $0.008-4 \mathrm{~Hz}$, respectively, during observations 1, 2, 6, 8, and 9 (top-left to bottom-right) with the best-fit model (solid line) and the model components (dotted line). The results of the fitting are shown in Table 2 and are discussed in the text.

As it is a common procedure in observational studies of NS systems, we used empirical models to describe the X-ray spectra. To compare our results with previous studies we used the same (or very similar) spectral components. To fit the spectral continuum we used a model consisting of photoelectric absorption (Balucinska-Church \& McCammon 1992; phabs in XSPEC) and a power-law with a high-energy exponential cutoff (cutoffpl). The absorption component was not used in Obs. 1-4 and 11 (we did not fit the data from Obs. 12 and 13 because of the lack of counts), since PCA spectra are not affected (i.e. at $E \geq 4.5 \mathrm{keV}$ ). In the case of XRT observations, with lower energy coverage (i.e. at $E \geq 1 \mathrm{keV}$ ) the absorption component was included in all our fits. This model provided significant wavy residuals in the whole spectra of all the observations with unacceptable chi-squared values $\left(\chi^{2} / v \approx 5-60\right.$; with $v$ being the number of degrees of freedom, i.e. d.o.f.).

The fits and the residuals of all the spectra were improved $\left(\chi^{2} / v \approx 1.5-6\right)$ by the addition of a cyclotron line scattering feature (CRSF; hereafter referred to as "cyclotron feature"). It was accounted for with a Gaussian absorption line (gabs). The use of this model component and/or the obtained line parameters are consistent with previous studies (Kreykenbohm et al. 2005; Tsygankov et al. 2006; Reig et al. 2006; Reig \& Nespoli 2013; Lutovinov et al. 2015). The parameters were left free in the HEXTE data and tied to those in the XRT and PCA data.

The fits still show significant residuals at $7-10 \mathrm{keV}$. This feature is known as the "10 keV feature", and its origin is uncertain (Coburn et al. 2002). We modelled it as a Gaussian absorption line (gabs), thus improving the fits substantially $\left(\chi^{2} / v \approx 1.0-1.2\right)$. In this case the parameters were left free in the PCA data and tied to those in the XRT and HEXTE data. The values obtained are within those expected, but with broader dispersion than those obtained for example for XTE J1946+274 by Müller et al. (2012).
Although beneath the spectral fits, there are still peaked positive residuals in the region $6-7 \mathrm{keV}$ of the PCA spectra. We accounted for them adding a Gaussian line profile (Gaussian) at $6.4-6.97 \mathrm{keV}$ in order to account for the $\mathrm{Fe} \mathrm{K}_{\alpha}$ fluorescence line. The PCA residuals flattened and we obtained the final fitting solution in this way.

To account for uncertainties in the absolute flux normalization between PCA and HEXTE we introduced a multiplicative constant that was fixed to 1 for the PCA and let to vary freely for HEXTE. In the case of XRT, PCA, and HEXTE data we fixed the multiplicative constant of PCA to 1 and let the rest vary freely. The most relevant results of this spectral analysis and the derived unabsorbed fluxes and luminosities are shown in Tables 3 and 4 and in Fig. 6.

In addition, we fitted the spectra obtained with Suzaku, during a much lower luminosity period (see Fig. 7 and Table 5). Spectra from XIS1, XIS3, and PIN were fitted in the 0.8-10 and $14-25 \mathrm{keV}$ energy ranges; the $1.6-2.3 \mathrm{keV}$ energy range was ignored owing to the uncertainties in calibration associated with the instrumental Si K edge ${ }^{16}$. The spectra were grouped to have at least 50 and 250 counts for the XIS and PIN, respectively. In this case a model consisting of photoelectric absorption (phabs) and a power law with an exponential high-energy cutoff of the type "highecut" was a much better description than "cutoffpl" (i.e. $\Delta \chi^{2}=15$ for the same number of d.o.f. and better residuals). With this model we obtained a statistics of $\chi^{2} / v=1.4$ and systematic residuals at $E<4 \mathrm{KeV}$, which we improved by adding a black-body spectrum with normalization proportional to the surface area (bbodyrad), obtaining fit statistics of $\chi^{2} / v=1.3$ (for the same number of d.o.f.). Because the normalization of the black-body component was not well constrained we tried to improve it and changed it by

\footnotetext{
${ }^{16}$ http://heasarc.gsfc.nasa.gov/docs/suzaku/analysis/ sical.html
} 
Table 3. Results from the RXTE spectral analysis only.

\begin{tabular}{llllll}
\hline \hline & Obs. 1 & Obs. 2 & Obs. 3 & Obs. 4 & Obs. 11 \\
\hline$E(1)_{\text {gabs }}$ & $7.6 \pm 2.8$ & $8.0 \pm 2.3$ & $9.0 \pm 0.5$ & $6.5 \pm 0.7$ & $5 \pm 2$ \\
$\sigma(1)$ & $4.6 \pm 1.6$ & $4.1 \pm 0.8$ & $3.3 \pm 0.6$ & $6 \pm 5$ & $7 \pm 3$ \\
$S(1)$ & $15 \pm 8$ & $9 \pm 7$ & $2.2 \pm 0.9$ & $70 \pm 40$ & $11 \pm 10$ \\
\hline$E(2)_{\text {gabs }}$ & $31.6 \pm 0.7$ & $31.3 \pm 0.8$ & $31.8 \pm 0.8$ & $29.7 \pm 1.2$ & $28.4 \pm 1.0$ \\
$\sigma(2)$ & $4.3 \pm 0.4$ & $4.6 \pm 0.4$ & $5.0 \pm 0.6$ & $4.1 \pm 0.8$ & $3.4 \pm 0.5$ \\
$S(2)$ & $17 \pm 3$ & $20 \pm 4$ & $23 \pm 5$ & $14 \pm 6$ & $8 \pm 3$ \\
\hline$E_{\text {Gauss }}$ & $6.3 \pm 0.5$ & $6.40 \pm 0.10$ & $6.9 \pm 1.5$ & $6.3 \pm 0.6$ & $5.7 \pm 0.6$ \\
$\sigma$ & $0.18 \pm 0.20$ & $0.22 \pm 0.20$ & $1.6 \pm 0.5$ & $0.5 \pm 0.4$ & $1.1 \pm 0.6$ \\
$N$ & $0.012 \pm 0.005$ & $0.009 \pm 0.004$ & - & $0.014 \pm 0.010$ & $0.02 \pm 0.01$ \\
\hline$\Gamma_{\text {pow }}$ & $-0.7 \pm 0.5$ & $-0.51 \pm 0.24$ & $-0.93 \pm 0.28$ & $-0.7 \pm 0.5$ & $-0.7 \pm 0.3$ \\
$E_{\text {cutoff }}$ & $5.7 \pm 0.6$ & $6.6 \pm 0.5$ & $5.7 \pm 0.6$ & $5.0 \pm 0.8$ & $5.68 \pm 0.15$ \\
\hline$F_{\mathrm{X}}$ & $10 \pm 4$ & $8 \pm 4$ & $4.7 \pm 0.1$ & $4.5 \pm 0.3$ & $1.3 \pm 0.7$ \\
$L_{\mathrm{X}}$ & $5.9 \pm 2.4$ & $4.7 \pm 2.4$ & $2.8 \pm 0.3$ & $2.7 \pm 0.3$ & $0.8 \pm 0.4$ \\
\hline$C_{\text {PCA }}$ & 1 & 1 & 1 & 1 & 1 \\
$C_{\text {HEXTE }}$ & $0.50 \pm 0.05$ & $0.45 \pm 0.07$ & $1.3 \pm 0.3$ & $1.5 \pm 0.3$ & $0.40 \pm 0.07$ \\
\hline$\chi^{2} / v$ & $0.83(43 / 52)$ & $0.90(47 / 52)$ & $1.1(56 / 52)$ & $1.2(64 / 52)$ & $1.1(56 / 52)$ \\
\hline
\end{tabular}

Notes. Model used: constant $\times$ gabs $\times$ gabs (cutoffpl + Gaussian) for the $4.5-60 \mathrm{keV}$ RXTE spectra. Centroid, line width, normalization of the Gaussian line, flux, and luminosity in units of $\mathrm{keV}, \mathrm{cm}^{-2} \mathrm{~s}^{-1}, 10^{-9} \mathrm{erg} \mathrm{s}^{-1} \mathrm{~cm}^{-2}$, and $10^{37} \mathrm{erg} \mathrm{s} \mathrm{s}^{-1}$, respectively. Errors are $68 \%$ confidence errors.

Table 4. Results from the Swift + RXTE spectral analysis.

\begin{tabular}{lcccccc}
\hline \hline & Obs. 5 & Obs. 6 & Obs. & Obs. 8 & Obs. 9 & Obs. 10 \\
\hline$N_{\mathrm{H}}\left(\times 10^{22}\right)\left(\mathrm{cm}^{-2}\right)$ & $1.01 \pm 0.08$ & $0.74 \pm 0.07$ & $0.84 \pm 0.07$ & $0.80 \pm 0.13$ & $0.77 \pm 0.07$ & $0.93 \pm 0.12$ \\
$E(1)_{\text {gabs }}(\mathrm{keV})$ & $4.1 \pm 1.6$ & $7.8 \pm 0.7$ & $8.5 \pm 0.5$ & $7.0 \pm 1.0$ & $7.2 \pm 0.6$ & $8.86 \pm 0.20$ \\
$\sigma(1)(\mathrm{keV})$ & $6.6_{-0.5}^{+1.7}$ & $4.3 \pm 0.7$ & $3.0 \pm 0.8$ & $5.1 \pm 1.4$ & $5.5 \pm 0.6$ & $7 \pm 3$ \\
Strength $(1)$ & $18_{-5}^{+13}$ & $12 \pm 4$ & $1.9_{-0.4}^{+1.7}$ & $28 \pm 14$ & $15 \pm 5$ & $60 \pm 30$ \\
\hline$E(2)_{\text {gabs }}(\mathrm{keV})$ & $30.8 \pm 0.8$ & $29.3 \pm 1.1$ & $26.8 \pm 0.9$ & $30.0 \pm 1.4$ & $30.0 \pm 2.0$ & $27.5 \pm 2.3$ \\
$\sigma(2)(\mathrm{keV})$ & $4.7 \pm 0.5$ & $3.4 \pm 0.4$ & $2.7 \pm 0.5$ & $4.0 \pm 0.7$ & $3.5 \pm 0.5$ & $2.15 \pm 1.0$ \\
Strength $(2)$ & $19 \pm 4$ & $14 \pm 4$ & $6.0 \pm 2.0$ & $12 \pm 4$ & $16 \pm 9$ & $10 \pm 8$ \\
\hline$E_{\text {Gauss }}(\mathrm{keV})$ & $6.56 \pm 0.16$ & $6.23 \pm 0.20$ & $6.23 \pm 0.19$ & $6.15 \pm 0.4$ & $6.5 \pm 0.5$ & $6.5 \pm 0.8$ \\
$\sigma(\mathrm{keV})$ & $0.004_{-0.003}^{+0.23}$ & $0.4 \pm 0.3$ & $0.34 \pm 0.20$ & $1.25 \pm 0.25$ & $0.003 \pm 0.003$ & $1.4 \pm 0.9$ \\
$N\left(\mathrm{~cm}^{-2} \mathrm{~s}^{-1}\right)$ & $0.55 \pm 0.10$ & $0.007 \pm 0.004$ & $0.0013 \pm 0.0010$ & $0.05 \pm 0.04$ & $0.0012 \pm 0.0007$ & $0.07 \pm 0.05$ \\
\hline$\Gamma_{\text {pow. }}$ & $-0.02 \pm 0.21$ & $-0.27 \pm 0.12$ & $0.05 \pm 0.10$ & $-0.20 \pm 0.20$ & $-0.49 \pm 0.10$ & $0.0 \pm 0.4$ \\
$E_{\text {cutoff }}(\mathrm{keV})$ & $5.4 \pm 0.4$ & $5.8 \pm 0.7$ & $10.3 \pm 1.6$ & $6.0 \pm 0.5$ & $5.2 \pm 0.7$ & $7 \pm 4$ \\
\hline$F_{\mathrm{X}}{ }^{1}\left(\times 10^{-9} \mathrm{erg} \mathrm{s}^{-1} \mathrm{~cm}^{-2}\right)$ & $4.3 \pm 0.4$ & $6.5 \pm 0.6$ & $6.3 \pm 0.6$ & $6.2 \pm 0.6$ & $3.3 \pm 0.3$ & $3.2 \pm 0.3$ \\
$L_{\mathrm{X}}\left(\times 10^{37} \mathrm{erg} \mathrm{s}^{-1}\right)$ & $2.5 \pm 0.3$ & $3.8 \pm 0.4$ & $3.8 \pm 0.4$ & $3.7 \pm 0.4$ & $2.0 \pm 0.2$ & $1.9 \pm 0.3$ \\
\hline$C_{\text {PCA }}$ & 1 & 1 & 1 & 1 & 1 \\
$C_{\mathrm{HEXTE}}$ & $1.43 \pm 0.20$ & $0.45 \pm 0.15$ & $0.11 \pm 0.05$ & $0.33 \pm 0.06$ & $0.61 \pm 0.15$ & $0.26 \pm 0.17$ \\
$C_{\text {XRT }}$ & $0.87 \pm 0.08$ & $0.39 \pm 0.03$ & $0.39 \pm 0.03$ & $0.29 \pm 0.03$ & $0.41 \pm 0.03$ & $0.48 \pm 0.05$ \\
\hline$\chi^{2} / v$ & $0.93(182 / 195)$ & $0.97(152 / 156)$ & $1.13(183 / 161)$ & $0.87(97 / 112)$ & $0.94(97 / 103)$ & $1.02(99 / 97)$ \\
\hline
\end{tabular}

Notes. Model used: phabs $\times$ constant $\times$ gabs $\times$ gabs (cutoffpl + Gaussian) for the $1-60 \mathrm{keV}$ Swift + RXTE spectra. Errors are $68 \%$ confidence errors.

an emission component describing an accretion disc consisting of multiple black-body components (diskbb; Mitsuda et al. 1984; Makishima et al. 1986). We obtained very similar fit statistics (see Table 5). A plausible origin for the first model component (bbodyrad) is the NS surface or perhaps the boundary layer (if present). Low-level accretion onto the surface of a neutron star can indeed produce a black-body-like spectrum (Zampieri et al. 1995). Still, a disc origin cannot be ruled out since it was also possible to fit the data with a multicolor disc black body. Similar results for a set of low-accreting NS have recently been obtained (Armas Padilla et al. 2013), but the temperatures found are $k T \approx=0.5-0.7 \mathrm{keV}$, thus much lower than in our case $(k T \approx=2.4 \pm 0.9 \mathrm{keV})$. The higher value we obtain might be indicative of the much higher accretion rate in our case.
Nevertheless, with the current data it is not possible to determine which model component is better able to describe the data.

To account for the uncertainties in the absolute flux normalization between XIS1, XIS3, and PIN we introduced a multiplicative constant that was fixed to 1 for XIS1 and left to vary freely for XIS3 and PIN. The most relevant results of this spectral analysis and the derived unabsorbed fluxes and luminosities are shown in Table 5.

\section{Discussion}

\subsection{Long-term Be-disc/neutron star interaction}

From the long-term observations of $\mathrm{V} 0332+53$ we can see that $\mathrm{V} 0332+53$ spends most of its time in the optical brightening 


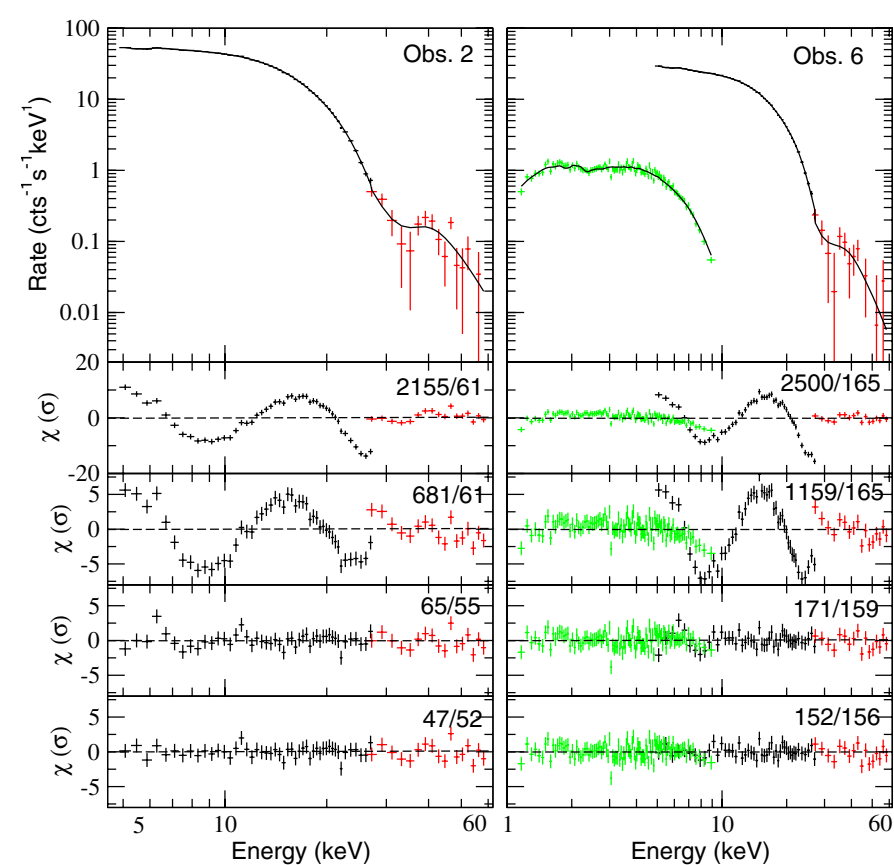

Fig. 6. Upper panels, left and right: two example spectra of V $0332+53$. Obs. 2 is one of the two observations with the highest count rates while Obs. 6 is the data set with the highest count rate with all the instruments available (black, red, and green correspond to PCA, HEXTE, and XRT, respectively). Lower panels: behaviour of the residuals when adding the different components one by one. The number in the right corner of each panel indicates the best-fit statistics ( $\chi^{2} /$ d.o.f.). From upper to lower panels: 1) best fit using the cutoffpl model component only (i.e. continuum model); 2) residuals after adding the $\approx 10 \mathrm{keV}$ absorption feature to the continuum model; 3 ) residuals after adding the cyclotron feature; 4) residuals after adding the $\mathrm{Fe} \mathrm{K}_{\alpha}$ line to the model (and final residuals of the model). The same vertical axis is used in both panels.

Table 5. Results from the Suzaku spectral analysis.

\begin{tabular}{lc}
\hline \hline & Obs. 14 \\
\hline$N_{\mathrm{H}}\left(\times 10^{22}\right)\left(\mathrm{cm}^{-2}\right)$ & $1.2 \pm 0.1$ \\
$k T_{\mathrm{bb}}(\mathrm{keV})$ & $2.4 \pm 0.9$ \\
$N_{\mathrm{bb}}$ & $0.011 \pm 0.007$ \\
\hline$\Gamma_{\text {pow. }}(\mathrm{keV})$ & $-1.3 \pm 1.6$ \\
$E_{\text {cutoff }}(\mathrm{keV})$ & $15-190$ \\
$E_{\mathrm{f}}(\mathrm{keV})$ & $1.0 \pm 1.0$ \\
\hline$F_{\mathrm{X}}\left(\times 10^{-11}\right)\left(\mathrm{erg} \mathrm{s}^{-1} \mathrm{~cm}^{-2}\right)$ & $2.4 \pm 0.9$ \\
$L_{\mathrm{X}}\left(\times 10^{35}\right)\left(\mathrm{erg} \mathrm{s}^{-1}\right)$ & $1.4 \pm 0.5$ \\
\hline$C_{\mathrm{XIS} 1}$ & 1 \\
$C_{\mathrm{XIS} 3}$ & $1.35 \pm 0.04$ \\
$C_{\mathrm{PIN}}$ & $0.4 \pm 0.3$ \\
\hline$\chi^{2} / v$ & $1.35(221 / 163)$ \\
\hline
\end{tabular}

Notes. Model used: phabs $\times$ constant $\times$ highecut $($ diskbb + powerlaw) for the $0.8-30 \mathrm{keV}$ Suzaku spectrum. Errors are $68 \%$ confidence errors.

phases (Fig. 2). The most significant phase is the period between 1993-2005. It started approximately four years after the giant outburst that the system exhibited in 1989 and peaked simultaneously with the X-ray maximum in 2004. All of the optical brightening episodes of BQ Cam are accompanied by X-ray activities. It is important to note that the brightness of the optical companion decreases after the X-ray outbursts. This behaviour

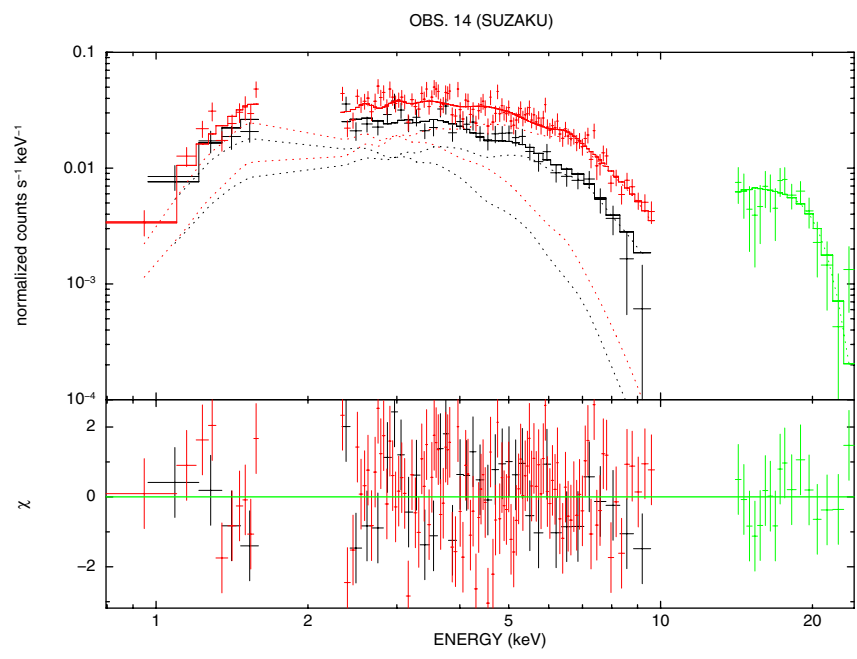

Fig. 7. Energy spectrum from V $0332+53$ during Obs. 14 with Suzaku (see Table 5 for the observation details and the results of the spectral analysis). Black, red, and green correspond to XIS1, XIS3, and PIN, respectively.

can be easily explained by the weakening of the decretion disc during the mass transfer to the NS.

Having a moderate eccentricity of $e=0.31$ with a short orbital period of $P_{\text {orb }}=34.25 \mathrm{~d}, \mathrm{~V} 0332+53$ is one of the BeXBs for which the effect of the truncation exerted by the NS is likely to be observed. Owing to the small orbit of the companion, the decretion disc cannot expand very much. Thus, it is truncated at a radius smaller than the critical Roche radius. Since the amount of material supported by the Be star to the disc is accumulated over time, the increase in the optical brightness can be understood in this way. However the accumulation of the material particularly at the outer part of the disc makes these regions become optically thick. According to theory by either the radiation-driving warping or a global density wave, the outer part of the disc is strongly elongated (Okazaki \& Negueruela 2001). In general, the existence of the global density waves in the decretion disc are observationally supported by the $V / R$ (i.e. the ratio of the peak in the $V$ and $R$ color of the $\mathrm{H}_{\alpha}$ line, respectively) variations seen in the emission line profiles. Although the line profile variations of V $0332+53$ were observed (Negueruela et al. 1998) during the period $1990-1991, \mathrm{H}_{\alpha}$ lines are always seen in nearly symmetric single-peaked emissions without any significant variations for the time interval 2006-2014. Therefore, we do not have any evidence to support the idea that perturbations in the disc or $V / R$ variations occurred.

\subsection{X-ray behaviour}

\subsubsection{Spectral evolution}

From the RXTE and Swift observations reported in this work we see a decrease in the X-ray luminosity of V $0332+53$ during the final part of the intermediate-luminosity 2008 outburst. Assuming a $7 \mathrm{kpc}$ distance (Negueruela et al. 1999), the luminosity gradually dropped from $\sim 6$ to $\sim 1 \times 10^{37} \mathrm{erg} \mathrm{s}^{-1}$ in the $5-60 \mathrm{keV}$ energy range from Obs. 1-11. The luminosities agree with those obtained during the decay phase of the 2005 outburst (Mowlavi et al. 2006). Finally, during the Suzaku observation (Obs. 14), we obtained the lowest luminosity $\left(\sim 1 \times 10^{35} \mathrm{erg} \mathrm{s}^{-1}\right)$.

A recent study of $\mathrm{V} 0332+53$ during giant outbursts at different luminosities has provided crucial insights into the most frequent states of V $0332+53$ (Reig \& Nespoli 2013). Their 


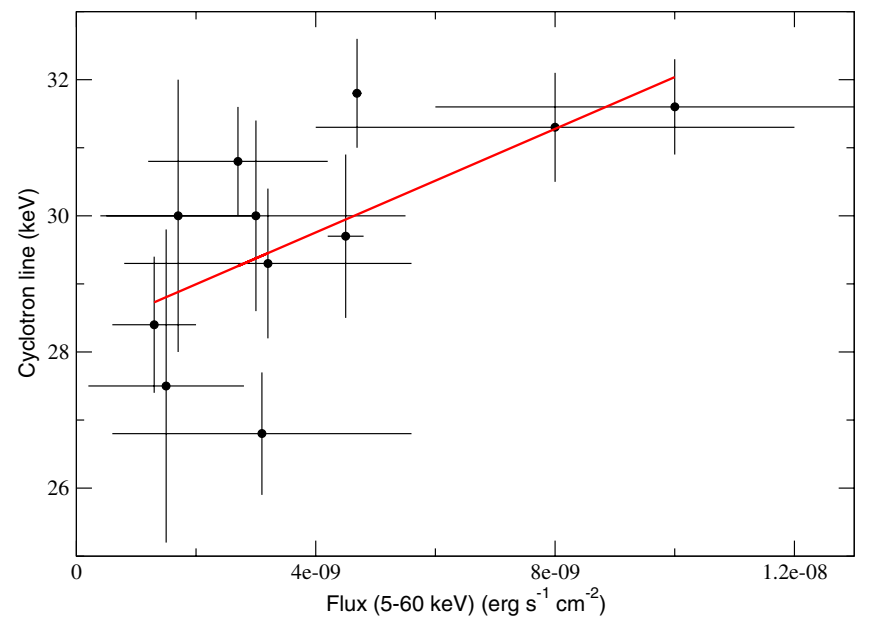

Fig. 8. Flux in the $5-60 \mathrm{keV}$ energy range versus the cyclotron line energy centre for Obs. 1-11. See Supplementary material Table A.3 for the list of unrounded values used.

Fig. 2 shows the existence of two separate regimes (the highluminosity diagonal branch and the low-luminosity horizontal branch). They are separated by a critical luminosity $\left(L_{\text {crit }} \approx\right.$ $1-4 \times 10^{37} \mathrm{erg} \mathrm{s}^{-1}$ ), which corresponds to the range of luminosities reported in our work during Obs. 1-11. Both regimes/states are clearly well populated by X-ray observations, while observations around the critical luminosity are scarce.

The spectral variability of $\mathrm{V} 0332+53$ has been carefully studied by different authors, confirming the softening of V $0332+53$ as the flux decreased. Baykal et al. (2007) suggested that this type of spectral softening with decreasing flux was mainly a consequence of mass accretion rate change. In particular, Reig et al. (2010, and references therein) explained that increased mass accretion rates are expected to result in harder $\mathrm{X}$-ray spectra due to Comptonization processes. In our work we see the same trend of photon index versus flux.

The values previously obtained for the energy centre of the cyclotron line have shown a strong anti-correlation with luminosity in V 0332+53 (Tsygankov et al. 2006, 2010; Mowlavi et al. 2006; Reig \& Nespoli 2013) during giant outbursts. Somewhat weaker anti-correlation has been found in 4U 0115+63 (Mihara et al. 2004; Nakajima et al. 2006) and no correlation in 1A 0535+262 (Caballero et al. 2007). In our work a positive correlation of the line energy centre with luminosity is observed (see Fig. 8). The correlation coefficient of the fit is +0.64 (i.e. null two-tailed probability of 0.03 ). This kind of behaviour has also been observed in A $0535+262$ during an intermediate-luminosity outburst $\left(L_{X}=4 \times 10^{37} \mathrm{erg} \mathrm{s}^{-1}\right.$; Sartore et al. 2015). We note that Reig \& Nespoli (2013) have already pointed out a tentative positive correlation for the latter source. This effect appears to be a natural consequence of accretion onto $\mathrm{X}$-ray pulsars with the strongest magnetic fields in the subcritical regime (Mushtukov et al. 2015). The values we obtain for the width of the cyclotron line are all $\approx 4 \mathrm{keV}$ and are in contrast to the values obtained during high flux states $(6-8 \mathrm{keV}$; Tsygankov et al. 2010), but in full agreement with those obtained in intermediate flux states (4 keV; Mowlavi et al. 2006).

\subsubsection{Quasi periodic oscillations}

We find that the PDS of V $0332+53$ during our observations are best described by band-limited noise plus QPO noise during the latest observations. The QPO noise consists of a component at a frequency of $\approx 0.22-0.23 \mathrm{~Hz}$. This feature has also been reported in the $2-60 \mathrm{keV}$ RXTE-PCA PDSs obtained during the 2004-2005 outburst decay (Qu et al. 2005). They found a QPO at $\approx 0.22 \mathrm{~Hz}$ in addition to a narrow peak (i.e. pulsation from the pulsar) close to that frequency $(\approx 0.23 \mathrm{~Hz})$. The QPO was detected when the luminosity was at a mid-flux level of the outburst, or even lower. The rms strength of this QPO also amounts to a few per cent in that work. The $0.22 \mathrm{~Hz}$ QPO was observed around the spin frequency in both the INTEGRAL (Mowlavi et al. 2006) and RXTE data during the 2005 outburst decline $(\mathrm{Qu}$ et al. 2005; Reig et al. 2006). In addition, Takeshima et al. (1994) discovered another QPO with a centroid frequency of $\approx 0.05 \mathrm{~Hz}$ and a relative rms amplitude of $\approx 5 \%$ in the $2.3-37.2 \mathrm{keV}$ data from the 1989 outburst of $\mathrm{V} 0332+53$. We do not find any signal of the QPO at $\approx 0.05 \mathrm{~Hz}$, although we find the presence of a broad component at around that frequency (which may eventually turn into peak noise, i.e. QPO). The QPO detected in V $0332+53$ at $\approx 0.22 \mathrm{~Hz}$ constitutes the first detection of a QPO riding on the spin frequency of a NS and points to a strong coupling between the periodic (i.e. the pulses) and the red-noise (i.e. broad-band QPO signal) components (Qu et al. 2005).

The existence of a QPO signal with a similar frequency to the spin of the NS suggests that they are related in some way. One model to explain this coincidence was given by Lazzati \& Stella (1997) and Burderi et al. (1997). They proposed that the QPO originates in the magnetosphere around the NS. Additionally, they argued that the accretion flow is inhomogeneous near the surface of the NS, and that random shots are characterized by an arbitrary degree of modulation. The combination of rotation and radiative transfer effects should therefore produce a periodic modulation of the shots similar to that of any continuum X-ray emission from the polar caps. They proposed that a coupling between the periodic and red-noise variability should be frequently present in X-ray pulsars.

In our case and in contrast to previous observations during giant flares (Reig et al. 2006; Reig 2008) the QPO signal appears during the lowest of the measured fluxes $\left(L_{\mathrm{X}} \leq 4 \times 10^{37} \mathrm{erg} \mathrm{s}^{-1}\right)$. If the QPO has an origin on the magnetosphere of the NS (and is not an artefact from our observations), it should be more visible when the accretion column is more tenuous (or even absent). In summary, it looks as if we might be witnessing the innermost parts closest to the magnetosphere surroundings during our latest observations, i.e. Obs. 6-11.

\section{Summary and conclusions}

In this paper we present a multiwavelength study of the Be/X-ray binary system $\mathrm{V} 0332+53$ with the main goal of studying its transient behaviour during its intermediate luminosity $\mathrm{X}$-ray outburst in 2008 . After being $\approx 3.5 \mathrm{yr}$ in quiescence in X-rays, the system underwent a new active period. A new X-ray outburst was detected around 17 October 2008, roughly six days before the periastron passage of the NS. We note that the optical companion entered the brightening state nearly $1.5 \mathrm{yr}$ before the X-ray outburst.

$\mathrm{V} 0332+53$ was in quiescence until the optical magnitude approached a peak value of $V=15.2 \mathrm{mag}$ at the end of 2009, indicating the beginning of another mass ejection event. In November 2009 a series of five small Type I periodic outbursts was observed. The X-ray activities finished by the end of May 2010 while the optical magnitude was still fading. It ceased around August 2011 for about $\approx 3.5$ yr. Our recent data shows that a mass ejection event is currently taking place, exhibiting an increase of $\approx 0.6$ in the $\mathrm{V}$ mag. It probably started around 
2013 and peaked in June 2015 when a new (Type II) X-ray outburst was detected. This is similar to those observed during the brightening episodes that occurred for example in 1983 and in 2004, but higher than those in 2008 and 2009. Our only IR measurement from December 2014 also showed an unusually bright Be companion.

The broad-band 1-60 keV X-ray spectra of the NS during the decay of the 2008 outburst were all well fitted with the standard cutoffpl phenomenological model, enhanced by a narrow iron $\mathrm{K}_{\alpha}$ fluorescence line at $6.4 \mathrm{keV}$ together with a cyclotron line scattering feature at $\approx 30 \mathrm{keV}$. Our study confirms the softening of $\mathrm{V} 0332+53$ as the flux decreased. During our lowest flux observation made with Suzaku $\left(L_{X}=10^{35} \mathrm{erg} \mathrm{s}^{-1}\right)$ in 2010 , compatible with $\mathrm{V} 0332+53$ being at a level slightly above quiescence, we detected a very soft spectrum that could be accurately described by adding one or more soft black-body component(s).

We tentatively see an increase of the cyclotron line energy with increasing flux. If confirmed with better observations, this might constitute the first detection of a positive correlation of the cyclotron line energy versus luminosity in $\mathrm{V} 0332+53$. Regarding the fast aperiodic timing properties, we detect a QPO at $227 \pm 9 \mathrm{mHz}$ during the lowest luminosities. This might indicate that the inner regions surrounding the magnetosphere are more visible during the lowest flux stages. Owing to their low significance, we advise that longer and better observations with current and/or future X-ray satellites are needed in order to confirm or discard these results.

Acknowledgements. We thank the anonymous referee and S. Campana for helpful comments. We also thank K. Page and the Swift team at Leicester (UK), A. J. Castro-Tirado (on behalf of the BOOTES collaboration), I. E. Papadakis and J. Garcia-Rojas for helpful discussions and insights, and for making part of these observations possible. R.H. acknowledges GA CR grant 13-33324S. M.C.G. acknowledges support by the European social fund within the framework of realizing the project "Support of inter-sectoral mobility and quality enhancement of research teams at Czech Technical University in Prague", CZ.1.07/2.3.00/30.0034. This research has made use of the General High-energy Aperiodic Timing Software (GHATS) package developed by T.M. Belloni at INAF-Osservatorio Astronomico di Brera.

\section{References}

Argyle, R. W., Kodaira, K., Bernacca, P. L., et al. 1983, IAU Circ., 3897, 2 Armas Padilla, M., Degenaar, N., \& Wijnands, R. 2013, MNRAS, 434, 1586 Arnaud, K. A. 1996, in Astronomical Data Analysis Software and Systems V, eds. G. H. Jacoby, \& J. Barnes, ASP Conf. Ser., 101, 17

Balucinska-Church, M., \& McCammon, D. 1992, ApJ, 400, 699

Baykal, A., Inam, S. Ç., Stark, M. J., et al. 2007, MNRAS, 374, 1108

Bernacca, P. L., Iijima, T., \& Stagni, R. 1984, A\&A, 132, 8

Bertin, E., \& Arnouts, S. 1996, A\&AS, 117, 393

Burderi, L., Robba, N. R., La Barbera, N., et al. 1997, ApJ, 481, 943

Caballero, I., Kretschmar, P., Santangelo, A., et al. 2007, A\&A, 465, 21

Camero, A., Zurita, C., Gutiérrez-Soto, J., et al. 2014, A\&A, 568, A115

Camero-Arranz, A., Finger, M. H., Ikhsanov, N. R., et al. 2010, ApJ, 708, 1500

Camero-Arranz, A., Caballero-Garcia, M. D., Ozbey-Arabaci, M., et al. 2015, ATel, 7682, 1

Coburn, W., Heindl, W. A., Rothschild, R. E., et al. 2002, ApJ, 580, 394

Coburn, W., Kretschmar, P., Kreykenbohm, I., et al. 2005, ATel, 381, 1
Coe, M. J., Payne, B. J., Hanson, C. G., et al. 1987, MNRAS, 226, 455 Corbet, R. H. D., Charles, P. A., \& van der Klis, M. 1986, A\&A, 162, 117 Evans, P. A., Beardmore, A. P., Page, K. L., et al. 2009, MNRAS, 397, 1177

Finger, M. H., Beklen, E., Narayana Bhat, P., et al. 2009, Fermi Symp., eConf Proc. C091122 [arXiv: 0912.3847]

Frank, J., King, A., \& Raine, D. J. 2002, Accretion Power in Astrophysics, 3rd edn. (Cambridge, UK: Cambridge University Press)

Gehrels, N.,Chincarini, G.,Giommi, P., et al. 2004, ApJ, 611, 1005

Giménez-García, A., Torrejón, J. M., Eikmann, W., et al. 2015, A\&A, 576, A108

Goranskii, V. P. 2001, Astron. Lett., 27, 516

Goranskij, V., \& Barsukova, E. 2004, ATel, 245, 1

Goranskij, V. P., Barsukova, E. A., \& Valeev, A. F. 2010, ATel, 2381, 1

Gruber, D. E., Blanco, P. R., Heindl, W. A., et al. 1996, A\&AS, 120, 641

Hanuschik, R. W. 1989, Ap\&SS, 161, 61

Honeycutt, R. K., \& Schlegel, E. M. 1985, PASP, 97, 300

Iye, M., \& Kodaira, K. 1985, PASP, 97, 1186

Jahoda, K., Swank, J. H., Giles, A. B., et al. 1996, SPIE Conf. Ser., 2808, 59

Kaur, R., Kumar, B., Paul, B., et al. 2008, ATel, 1807, 1

Kiziloglu, U., Kiziloglu, N., Baykal, A., et al. 2008, IBVS, 5865, 1

Kodaira, K., Nishimura, S., Kondo, M., et al. 1985, PASJ, 37, 97

Koyama, K., Tsunemi, H., Dotani, T., et al. 2007, PASJ, 59, 23

Kreykenbohm, I., Mowlavi, N., Produit, N., et al. 2005, A\&A, 433, 45

Krimm, H. A., Barthelmy, S. D., Baumgartner, W., et al. 2008, ATel, 1792, 1

Krimm, H. A., Barthelmy, S. D., Baumgartner, W., et al. 2009, ATel, 2319, 1

Krimm, H. A., Holland, S. T., Corbet, R. H. D., et al. 2013, ApJS, 209, 14

Lazzati, D., \& Stella, L. 1997, ApJ, 476, 267

Leahy, D. A., Elsner, R. F., \& Weisskopf, M. C. 1983, ApJ, 272, 256

Lutovinov, A. A., Tsygankov, S. S., Suleimanov, V. F., et al. 2015, MNRAS, 448,2175

Makishima, K., Maejima, Y., Mitsuda, K., et al. 1986, ApJ, 308, 635

Makishima, K., Mihara, T., Ishida, M., et al. 1990, ApJ, 365, 59

Masetti, N., Orlandini, M., Marinoni, S., et al. 2005, ATel, 388, 1

Mas-Hesse, J. M., Giménez, A., Culhane, J. L., et al. 2003, A\&A, 411, L261

Mihara, T., Makishima, K., \& Nagase, F. 2004, ApJ, 610, 390

Mitsuda, K., Inoue, H., Koyama, K., et al. 1984, PASJ, 36, 741

Mitsuda, K., Bautz, M., Inoue, H., et al. 2007, PASJ, 59, 1

Mowlavi, N., Kreykenbohm, I., Shaw, S. E., et al. 2006, A\&A, 451, 187

Müller, S., Kühnel, M., Caballero, I., et al. 2012, A\&A, 546, A125

Mushtukov, A. A., Suleimanov, V. F., Tsygankov, S. S., \& Poutanen, J. 2015, MNRAS, 447, 1847

Nakajima, M., Mihara, T., Makishima, K., Niko, H., et al. 2006, ApJ, 646, 1125

Nakajima, M., Sugizaki, M., Matsuoka, M., J., et al. 2010, ATel, 2427, 1

Negueruela, I., Reig, P., Coe, M. J., et al. 1998, MNRAS, 336, 251

Negueruela, I., Roche, P., Fabregat, J., et al. 1999, MNRAS, 307, 695

Okazaki, A. T., \& Negueruela, I. 2001, A\&A, 377, 161

Pottschmidt, K., Kreykenbohm, I., Wilms, J., et al. 2005, ApJ, 634, 97

Qu, J. L., Zhang, S., Song, L., M., et al. 2005, ApJ, 629, 33

Reig, P. 2008, A\&A, 489, 725

Reig, P., \& Nespoli, E. 2013, A\&A, 551, A1

Reig, P., Martínez-Núñez, S., \& Reglero, V. 2006, A\&A, 449, 703

Reig, P., Słowikowska, A., Zezas, A., \& Blay, P. 2010, MNRAS, 401, 55

Sartore, N., Jourdain, E., \& Roques, J. P. 2015, ApJ, 806, 193

Stella, L., White, N. E., Davelaar, J., et al. 1985, ApJ, 288, 45

Takahashi, T., Abe, K., Endo, M., et al. 2007, PASJ, 59, 35

Takeshima, T., Dotani, T., Mitsuda, K., et al. 1994, ApJ, 436, 871

Terrell, J., \& Priedhorsky, W. C. 1984, ApJ, 285, 15

Tsygankov, S. S., Lutovinov, A. A., Churazov, E. M., et al. 2006, MNRAS, 371, 19

Tsygankov, S. S., Lutovinov, A. A., \& Serber, A. V. 2010, MNRAS, 401, 1628

Unger, S. J., Norton, A. J., Coe, M. J., et al. 1992, MNRAS, 256, 725

Williams, P. M., Brand, P. W. J. L., Bell Burnell, S. J., et al. 1983, IAU Circ.,

3904, 2

Winkler, C., Courvoisier, T. J.-L., Di Cocco, G., et al. 2003, A\&A, 411, L1

Zampieri, L., Turolla, R., Zane, S., et al. 1995, ApJ, 439, 849

Zhang, S., Qu, J.-L., Song, L.-M., et al. 2005, ApJ, 630, 65 


\section{Appendix A: Additional tables}

Table A.1. Optical photometric observations from the IAC80 telescope.

\begin{tabular}{lcccc}
\hline \hline MJD & $V$ & Error & $B$ & Error \\
\hline 56871.2368550198 & 15.460 & 0.013 & 17.037 & 0.071 \\
56993.9640124198 & 15.360 & 0.006 & 16.919 & 0.021 \\
57009.0723979599 & 15.373 & 0.005 & 17.006 & 0.013 \\
57026.9773657001 & 15.372 & 0.008 & 16.945 & 0.031 \\
57036.8705536202 & 15.373 & 0.005 & 16.967 & 0.013 \\
57054.0418117600 & 15.332 & 0.008 & 16.853 & 0.031 \\
57054.8470330602 & 15.364 & 0.006 & 16.955 & 0.024 \\
\hline
\end{tabular}

Table A.2. $\mathrm{H}_{\alpha}$ line EW and FWHM measurements of V 0332+53.

\begin{tabular}{|c|c|c|c|}
\hline$E$ & $\begin{array}{l}E W \\
(\AA) \\
\end{array}$ & $\begin{array}{c}F W H M \\
(\AA)\end{array}$ & 7 \\
\hline$\overline{006}$ & $238-6.21 \pm$ & $.18 \pm 0.38$ & TT15 \\
\hline & & & \\
\hline & & & 150 \\
\hline & & & \\
\hline 07 & $1-4.80$ & & 150 \\
\hline 007 & $2-3.82=$ & & TT150 \\
\hline & $0-5.2$ & & TT150 \\
\hline & & & \\
\hline & & & \\
\hline & 9 & & \\
\hline & & & \\
\hline & & & \\
\hline & & & \\
\hline & & & \\
\hline & & & \\
\hline & 7 & & \\
\hline & 55 & & \\
\hline & $4-4.7$ & & 150 \\
\hline & $4-5.4$ & & 50 \\
\hline & $4-4.2$ & & \\
\hline & $2-3$ & & 50 \\
\hline & $8-4$ & & 150 \\
\hline & $2-4.8$ & 7. & \\
\hline & $7-6$ & & 50 \\
\hline & 0 & 6.7 & \\
\hline & 0 & & \\
\hline & & & \\
\hline & 8 & & 50 \\
\hline & & & \\
\hline & -4 & & \\
\hline & 566 & & \\
\hline & $5-4.92$ & & \\
\hline & 240 & & \\
\hline & 45 & & \\
\hline
\end{tabular}

Notes. Negative values of EW indicate that the line is in emission.
Table A.3. Unrounded values from Table 3 used in Fig. 8.

\begin{tabular}{lll}
\hline \hline Obs. & $E(2)_{\text {gabs }}(\mathrm{keV})$ & $F_{X}\left(\mathrm{erg} \mathrm{s}^{-1} \mathrm{~cm}^{-2}\right)$ \\
\hline 1 & $31.6 \pm 0.7$ & $(1.0 \pm 0.4) \times 10^{-8}$ \\
2 & $31.3 \pm 0.8$ & $(0.8 \pm 0.4) \times 10^{-8}$ \\
3 & $31.8 \pm 0.8$ & $(4.70 \pm 0.10) \times 10^{-9}$ \\
4 & $29.7 \pm 1.2$ & $(4.5 \pm 0.3) \times 10^{-9}$ \\
5 & $30.8 \pm 0.8$ & $(2.7 \pm 1.5) \times 10^{-9}$ \\
6 & $29.3 \pm 1.1$ & $(3.2 \pm 2.4) \times 10^{-9}$ \\
7 & $26.8 \pm 0.9$ & $(3.1 \pm 2.5) \times 10^{-9}$ \\
8 & $30.0 \pm 1.4$ & $(3.0 \pm 2.5) \times 10^{-9}$ \\
9 & $30.0 \pm 2.0$ & $(1.7 \pm 1.3) \times 10^{-9}$ \\
10 & $27.5 \pm 2.3$ & $(1.5 \pm 1.3) \times 10^{-9}$ \\
11 & $28.4 \pm 1.0$ & $(1.3 \pm 0.7) \times 10^{-9}$ \\
\hline
\end{tabular}

Notes. Fluxes are in the 5-60 keV energy range. Errors are $68 \%$ confidence errors. 